\title{
Sublaminar bands: are they safe?
}

\author{
E. Polirsztok $\cdot$ M. Gavaret $\cdot$ T. Gsell $\cdot$ \\ I. Suprano $\cdot$ E. Choufani $\cdot$ G. Bollini $\cdot$ \\ Jean-Luc Jouve
}

Received: 21 March 2014/Revised: 23 September 2014/Accepted: 23 September 2014/Published online: 8 October 2014

(C) The Author(s) 2014. This article is published with open access at Springerlink.com

\begin{abstract}
Background Sublaminar bands (SB) are frequently used as implants in spine deviation correction.

Purpose Our purpose is to demonstrate their safety on a large series of patients.

Study design This is a retrospective study.

Methods Our department treated 378 spine deviations on children and adolescents via a hybrid posterior technique (lumbar screws, hook and thoracic SB). Each surgery was undertaken using anesthesiologic and a neurophysiologic monitoring using somatosensory evoked potentials (SSEP) and neurogenic mixed evoked potentials (NMEP). An alert was described as an amplitude decrease of $50 \%$ and/or a latency increase of $10 \%$. Data were analyzed using Student or Wilcoxon tests.
\end{abstract}

E. Polirsztok $(\bowtie) \cdot$ E. Choufani · G. Bollini

Pediatrics Orthopedic Surgery Department,

AP-HM La Timone Children's Hospital, 264 rue Saint-Pierre,

13385 Marseille Cedex 5, France

e-mail: evapolirsztok@gmail.com

M. Gavaret

Clinical Neurophysiologic Department,

AP-HM La Timone Hospital, Aix-Marseille University,

13444 Marseille Cedex 15, France

T. Gsell · I. Suprano

Anesthesiologic Department, AP-HM La Timone

Children's Hospital, 13385 Marseille Cedex 5, France

J.-L. Jouve

Pediatrics Orthopedic Surgery Department, AP-HM La

Timone Children's Hospital, Aix-Marseille University,

13385 Marseille Cedex 5, France

e-mail: Jean-luc.JOUVE@ap-hm.fr
Results We used 2,223 SB in 378 operative procedures. We described ten neurophysiologic alerts during the passage of the band under the lamina. There were no significative differences between the two groups concerning the age and the severity of the deformation $(p>0.05)$. These neurophysiologic alerts were associated with a dysautonomic trouble (hypertension and bradycardia). The lesional level was determined using a spinal electrode. In six cases, the responsible SB was removed. Three patients had postoperative neurologic deficiency $(0.8 \%)$ without complete recovery for one of them (localized incomplete sensitive deficiency). Within the group of 378 patients, 21 alerts were reported due to a screw or a hook, or during the correction manoeuver, without dysautonomic trouble.

Conclusion SB neurologic complications rate is as high as other implants' complication rate. Simultaneous hemodynamic and neurophysiologic change is an argument for vegetative response due to SB passage. Their optimal use requires a strict learning of their insertion under the lamina to be as less traumatic as possible. SB are as safe as any other spine implants.

Keywords Spine deviation correction - Children . Sublaminar band - Intra-operative monitoring . Anesthesiologic monitoring - Dysautonomic trouble

\section{Introduction}

Surgery of spine deviation correction on children and adults, whatever the etiology, has considerably improved over the past few years, thanks to new implant developments. Each implant has its advantages and drawbacks. Success depends on implantation location and surgical indication. 
We chose hybrid instrumentation, with lumbar pedicular screws, thoracic sublaminar bands and proximal bilaminar hooks.

Compared to other implants, sublaminar bands prove to be effective and have a good biomechanical tolerance [1]. They allow good correction in the frontal plane. They are particularly effective in the sagittal plane with better restoration of the thoracic kyphosis [2].

A polymeric band, the rigid end of which is shaped by the surgeon, is used during the operative procedure. The rigid curved end is introduced safely under the lamina. With a specific tool, bands are tensioned, step by step, pulling the vertebra by the lamina, up to the correction rod. The correction is progressive. Procedure times are significatively reduced.

We have been using sublaminar bands (SB) since 2006. Other publications have reported their efficacy in spine deviation correction $[3,4]$. Since then, we have found perioperative neurophysiologic alerts corresponding to the band passage under the lamina.

In this retrospective study, we analyze the occurrence of neurophysiologic alerts.

The study's main objective is to evaluate the frequency and severity of neurologic complications due to SB. Secondarily, we have analyzed risk factors, hemodynamic consequences and the management of peri-operative alerts.

\section{Materials and methods}

Study, population and inclusion criteria

In this monocentric retrospective study, we reviewed all cases of spine deviation correction surgery undertaken using hybrid instrumentation (thoracic sublaminar bands, lumbar pedicle screws, proximal hooks). Surgical procedures were carried out by different surgeons from the same orthopedic department, between January 2006 and July 2013.

All programmed procedures for scoliosis or kyphosis with intra-operative neurophysiologic monitoring were included.

\section{Surgical protocol}

The surgical procedure was a classical posterior skin incision, in a ventral decubitus, on a spine operative table or on scapular and pelvic blocks. Instrumentation levels were determined based on bending pre-operative X-rays. Pedicle screws were used for lumbar vertebra, up to the T10 level. For the arthrodesis, the proximal level was selected and we used a bilaminar hook system [5]. Between the proximal and distal levels, each vertebra was instrumented with a sublaminar band in the convexity of the deformation. The band was shaped manually by the surgeon to obtain an optimal rectangular shape, allowing its atraumatic introduction under the lamina. Then, the band was passed, making a loop, in a specific jaw, fixed on the correction rod. The deformity correction was obtained by pulling the band tight in a stepwise fashion, with a specific tool passed in the loop. In this manner, the vertebrae were pulled by the convexity up to the correction rod. They were pulled gradually, level by level, several times each in turn, until obtaining the correction expected on the pre-operative $\mathrm{X}$-ray bending. When the expected correction was obtained, the band was locked in the jaw.

Following the SFCR (the French Society of Spine Surgery) guidelines [6], we used intra-operative multimodal monitoring (SSEP, NMEP, D waves and pedicle screws test), performed by a trained neurophysiologist in the operative room. For all patients, we had a pre-operative SSEP record to refer to. During each alert, we followed the steps in the guidelines: anesthesiologic monitoring parameters were checked and then the alert was confronted by the surgical act. In case of persistent monitoring alert, the lesional level was determined using an epidural spinal electrode and the incriminated band was removed. For the neurophysiologic alerts unrelated to SB, deformity correction was released, or pedicle screw was replaced or removed. In case of persistent monitoring alert, the surgical procedure was stopped, all implants removed and a wakeup test was performed.

\section{Anesthesiological protocol}

Anesthesiologic induction was performed under standard monitoring (electrocardiogram, pulse oximeter and noninvasive arterial blood pressure monitor). Inhalational induction (sevoflurane, nitrous oxide) or intravenous induction was used. Two good caliber peripheral intravenous lines were put in. After orotracheal intubation, the implementation, or not, of an arterial line was left for the anesthesiologist to decide. Anesthesia was maintained using the intravenous line only (with propofol, remifentanil or sufentanil, cisatracurium). The respiratory circuit was cleansed, to facilitate the intra-operative neurophysiologic monitoring.

\section{Neurophysiologic monitoring protocol}

Neurophysiologic monitoring included intra-operative SSEP and NMEP recordings, supplemented since 2011, with pedicle screw test and D wave recordings. SSEP were obtained by stimulation of posterior tibialis nerves. 
Peripheral SSEP recordings are performed at the level of the internal popliteal sciatic nerves. A cortical recording is performed in $\mathrm{Cz}$, with regard to the primary somesthetic cortex of both the lower limbs. SSEP are relayed by NMEP as soon as the spinal epidural electrode is fitted at the proximal end of the instrumentation [7]. NMEP are obtained by stimulating the spinal cord through the electrode inserted by the surgical team. NMEP recordings are performed at the internal popliteal sciatic nerves, with subcutaneous needles.

$\mathrm{D}$ (direct) waves allow monitoring the motor pathways from the cortex to the level of the spinal electrode. D waves are obtained by transcranial electrical stimulation. The recording is spinal, generally at the level of T11 (or at the caudal part of the operating field).

For pedicle screw testing, when the patient is not under curarization, each pedicle screw is electrically stimulated with an increasing intensity from 5 to $30 \mathrm{~mA}$. Recordings are performed at the level of the lower limb muscles. The objective is to estimate the motor response threshold.
Data analysis

Epidemiologic data (age, sex, height) and spine deformity data (etiology, deformity reduction, Cobb angle) were analyzed.

Hemodynamic parameters (pulse, arterial blood pressure) were collected thanks to the peri-operative anesthesiologic diagram.

Surgical analyzed data included primary or secondary surgical procedure, number of sublaminar bands used and location of sublaminar band implantation.

Intra-operative neurophysiologic monitoring data were found on peri-operative reports and checked by the referent neurologist.

A monitoring alert was defined as a reduction of at least $50 \%$ of the amplitude and/or an increase of at least $50 \%$ of their latency, with or without post-operative neurologic deficiency. An alert was linked to SB if situated at the thoracic level, after lesional level determination by the spinal electrode, and without a link to other implants and/or
Table 1 The etiology of spine deformity managed between 2006 and 2013

\begin{tabular}{|c|c|c|c|}
\hline \multicolumn{2}{|c|}{ Etiology of spine deformities managed between 2006 and 2013} & \multirow{2}{*}{$\begin{array}{l}\begin{array}{l}\text { Mean Cobb } \\
\text { angle }\end{array} \\
52.1^{\circ}\end{array}$} & \multirow{2}{*}{$\begin{array}{l}\begin{array}{l}\text { Number of } \\
\text { used bands }\end{array} \\
1,629\end{array}$} \\
\hline Idiopathic scoliosis & 262 & & \\
\hline Secondary scoliosis & 100 & $60.5^{\circ}$ & 594 \\
\hline Malformative & 24 & $59^{\circ}$ & 91 \\
\hline Hemi-vertebra & 18 & $53,1^{\circ}$ & 73 \\
\hline Other malformations & 6 & $65^{\circ}$ & 18 \\
\hline Neurologic & 34 & $67.7^{\circ}$ & 177 \\
\hline Spina bifida & 3 & $84.6^{\circ}$ & 22 \\
\hline Medullary malformation & 9 & $61.15^{\circ}$ & 43 \\
\hline $\mathrm{CP}$ & 22 & $57^{\circ}$ & 112 \\
\hline Syndromic & 16 & $62^{\circ}$ & 63 \\
\hline Little & 1 & NR & 2 \\
\hline DiGeorge & 2 & $48^{\circ}$ & 5 \\
\hline Prader Willi & 3 & $67^{\circ}$ & 6 \\
\hline Marfan & 4 & $55^{\circ}$ & 17 \\
\hline Ellis Van Creuveld & 1 & $92^{\circ}$ & 1 \\
\hline Chromosomal anomaly/T21 & 3 & $64^{\circ}$ & 20 \\
\hline Klinefelter & 1 & $46^{\circ}$ & 6 \\
\hline Acromegaly & 1 & NR & 6 \\
\hline Bone dystrophy & 15 & $61.3^{\circ}$ & 86 \\
\hline Achondroplasia & 1 & NR & 5 \\
\hline NF1 & 10 & $64.6^{\circ}$ & 60 \\
\hline Other bone dystrophies & 4 & $58^{\circ}$ & 21 \\
\hline Myopathy & 2 & $60^{\circ}$ & 17 \\
\hline Post-tumoral & 4 & $68^{\circ}$ & 30 \\
\hline Post-surgical & 5 & $60.2^{\circ}$ & 37 \\
\hline Hyperkyphosis (Scheuermann) & 3 & $90^{\circ}$ & 21 \\
\hline \multirow[t]{2}{*}{ Secondary hyperkyphosis } & 13 & NR & 72 \\
\hline & 378 & $54.1^{\circ}$ & 2,223 \\
\hline
\end{tabular}


Table 2 Etiology of spine deformities presented by patients who had per-operative alerts

\begin{tabular}{lrlc}
\hline $\begin{array}{l}\text { Etiology of spine deformities presented by } \\
\text { patients who had pre-operative alert }\end{array}$ & $\begin{array}{l}\text { Mean Cobb } \\
\text { angle }\end{array}$ & $\begin{array}{l}\text { Number } \\
\text { of used } \\
\text { bands }\end{array}$ \\
\hline Idiopathic scoliosis & 5 & $51^{\circ}$ & 34 \\
Secondary scoliosis & 3 & $54^{\circ}$ & 17 \\
Hemi-vertebra & 1 & $70^{\circ}$ & 5 \\
Chondrodysplasia & 1 & $60^{\circ}$ & 5 \\
Mixed: thoracogenic and neurologic & 1 & $32^{\circ}$ & 7 \\
Hyperkyphosis (Scheuermann) & 1 & $100^{\circ}$ & 6 \\
Kyphosis T4T5 (spondylodiscitis) & 1 & $20^{\circ}$ & 8 \\
& 10 & $55.5^{\circ}$ & 65 \\
\hline
\end{tabular}

spine deformity correction. After each alert, the emergency surgical procedure (removal of a band, medullary decompressive procedure) and the evolution of intra-operative monitoring data were collected.

Data from the systematic post-operative neurologic clinical examination and the clinical examination at the follow-up were also collected.

\section{Statistics}

The parametric Student's test was used. We used the nonparametric Wilcoxon test in case the application conditions of Student's test were not respected. The test was significant if $p<0.05$.

\section{Results}

Between 2006 and 2013, in our department, 378 spine deformities were instrumented with hybrid instrumentation, by different surgeons, with intra-operative neurophysiologic monitoring.

Among these 378 patients, 262 had idiopathic scoliosis $(69.3 \%), 114$ secondary scoliosis (30.2\%) and 2 kyphosis $(0.5 \%)$ (Table 1$)$. This represents 2,223 sublaminar bands used, that is to say an average of 5.9 bands per surgical procedure.

The average age was 14 years (3-23 years old) at the time of surgery. There were 287 girls $(75.9 \%)$ and 91 boys (24\%).

Ten of the 378 patients presented a per-operative alert during the passage of a thoracic band $(2.1 \%$ of the 378 patients) (Table 2).

The average age of the patients was 14.2 years (10-19 years old) at the time of surgery. They were seven girls $(70 \%)$ and three boys (24\%).

Five of these ten patients presented an idiopathic scoliosis with an average Cobb angle of $51^{\circ}$. Three presented a secondary scoliosis with an average Cobb angle of $54^{\circ}$ (1 lumbar hemivertebra, 1 chondrodysplasia, 1 thoracogenic and neurologic scoliosis on a child with T21 and Fallot tetralogy). One presented a thoracic hyperkyphosis as a sequela of Scheuermann disease. The last one presented a hyperkyphosis as a sequela of a T4T5 spondylodiscitis.

The average Cobb angle for the ten children was $55.5^{\circ}$, that is to say an average angle comparable to the other 378 patients $\left(54.1^{\circ}\right)$.

We compared the 10 patients with the whole group of 378 patients to make sure that there were no significant signs that made them more likely to present a neurophysiologic and anesthesiologic alert. The application conditions of Student's test for this comparison were not respected, so we used the Wilcoxon test.

Table 3 Characteristics of the ten patients with intra-operative monitoring alerts

\begin{tabular}{|c|c|c|c|c|c|c|c|c|}
\hline & Age (years) & Date & Sex & Diagnosis & Apex & Intervention & $\begin{array}{l}\text { No. of used } \\
\text { bands }\end{array}$ & $\begin{array}{l}\text { No. of removed } \\
\text { bands }\end{array}$ \\
\hline 1 & 13 & $09 / 07$ & $\mathrm{~F}$ & AIS $60^{\circ}$ & $\mathrm{T} 8$ & PA T3L2 & 3 & 0 \\
\hline 2 & 13 & $04 / 11$ & $\mathrm{~F}$ & AIS $70^{\circ}$ & $\mathrm{T} 8$ & PA T1L3 & 8 & $2(\mathrm{~T} 9, \mathrm{~T} 10)$ \\
\hline 3 & 12 & 07/11 & $\mathrm{F}$ & AIS $42^{\circ}$ & Т9T10 & PA & 6 & 1 (T5) \\
\hline 4 & 17 & $09 / 12$ & M & AIS $40^{\circ}$ & T9T10 & PA T2L2 & 10 & $3(\mathrm{~T} 4, \mathrm{~T} 5, \mathrm{~T} 6)$ \\
\hline 5 & 13 & $10 / 12$ & $\mathrm{~F}$ & AIS $52^{\circ}$ & Т8Т9 & PA T2L2 & 7 & 0 \\
\hline 6 & 17 & $09 / 09$ & $\mathrm{~F}$ & Chondrodysplasia $60^{\circ}$ & $\mathrm{T} 7$ & PA & 5 & 5 \\
\hline 7 & 10 & $03 / 11$ & $\mathrm{~F}$ & Spondylodiscitis & T4T5 & PA & 8 & 1 \\
\hline 8 & 13 & $04 / 11$ & $\mathrm{~F}$ & Hemivertebra $70^{\circ}$ & $\mathrm{T} 11$ & PA & 5 & 0 \\
\hline 9 & 19 & $10 / 12$ & M & Kyphosis $100^{\circ}$ & T9 & SPO T5T12 and PA & 6 & $2(\mathrm{~T} 6, \mathrm{~T} 7)$ \\
\hline \multirow[t]{2}{*}{10} & 15 & $05 / 13$ & M & $\mathrm{T} 2132^{\circ}$ & $\mathrm{T} 10$ & PA & 7 & 1 (T4) \\
\hline & & & & & & & 65 & 15 \\
\hline
\end{tabular}

The lesional level was distinct at the apex level. In case of spontaneously resolved alert, sublaminar bands were not removed AIS adolescent idiopathic scoliosis, PA posterior arthrodesis, SPO Smith-Peterson osteotomy 


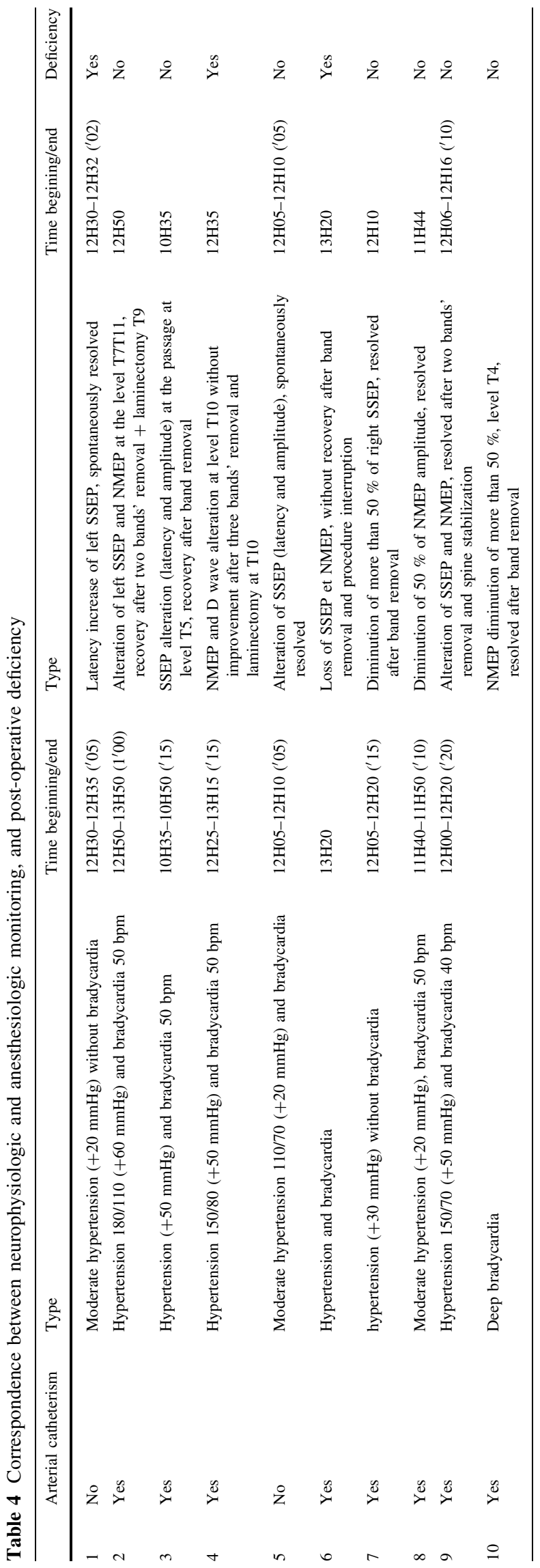

There was no significant difference between those two groups as far as age was concerned $(p=0.61)$, or for deformation severity (with comparison of the Cobb angle) $(p=0.91)$.

For one patient, of the ten-patient group, it was a second surgery, and for the other nine patients, it was a first-time procedure.

Sixty-five bands were passed on these 10 patients, an average of 6.5 bands per procedure. Out of these 65 bands, 30 were involved in neurophysiologic alerts and 15 were removed ( $0.67 \%$ of the 2,223 implants). Lesional levels identified did not always correspond to the apex vertebra (Table 3).

Three patients presented a post-operative neurologic deficiency, with progressive recovery, but it was incomplete for one of them, that is, showing a peri-operative neurologic complication rate of $0.8 \%$.

Patient number 1 presented a hyposensibility at the T10 level that spontaneously resolved within a few days. Patient number 4 presented a right leg pyramidal syndrome that was persistent 4 months later. A medullary injury was diagnosed on an immediate post-operative MRI. Perioperative NMEP remained diminished, and SSEP unchanged. Six months later, a suspended sensory deficiency at the level of the trunk persisted. Patient 6 presented errors in the pick-touch test and pallesthetic perturbations after surgery, which spontaneously resolved within a few days (Table 4).

In these alerts related to $\mathrm{SB}$, neurophysiologic monitoring alerts were concomitant with anesthesiologic monitoring alerts. Indeed, continued arterial blood pressure monitoring, by catheterism, highlights a hypertension increase associated with bradycardia, seen on usual anesthesiologic monitoring. Hemodynamic perturbations were found as long as neurophysiologic alerts lasted (Table 4).

Among the 378 monitored spine surgeries between 2006 and 2013, we noticed 21 other cases (10 idiopathic scoliosis, average age 14,1 years; and 11 secondary scoliosis, average age 14 years) of neurophysiologic alerts, which happened without any link to SB passage (3 in 2006, 4 in 2007, 9 in 2008, 2 in 2009, 2 in 2010, 1 in 2012). Seven alerts were due to a pedicular screw (1.8\% of the whole patients group), 11 occurred during the correction maneuver $(2.9 \%)$, 1 was secondary to hypotension ischemia $(0.26 \%), 1$ due to bleeding $(0.26 \%)$ and 1 during an osteotomy $(0.26 \%)$. For the 21 patients, no neurophysiologic alerts or neurologic post-operative deficiency was observed.

\section{Discussion}

We started using SB in 2006, the reason why our inclusion period started in 2006. All our operative cases are included 

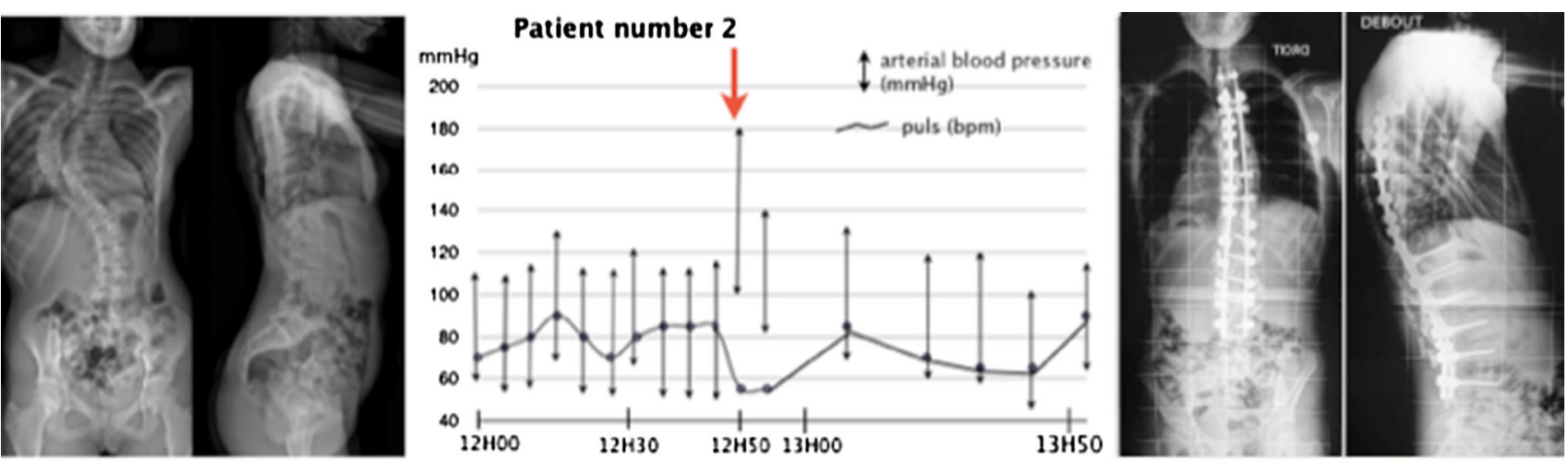

SSEP begining of surgery

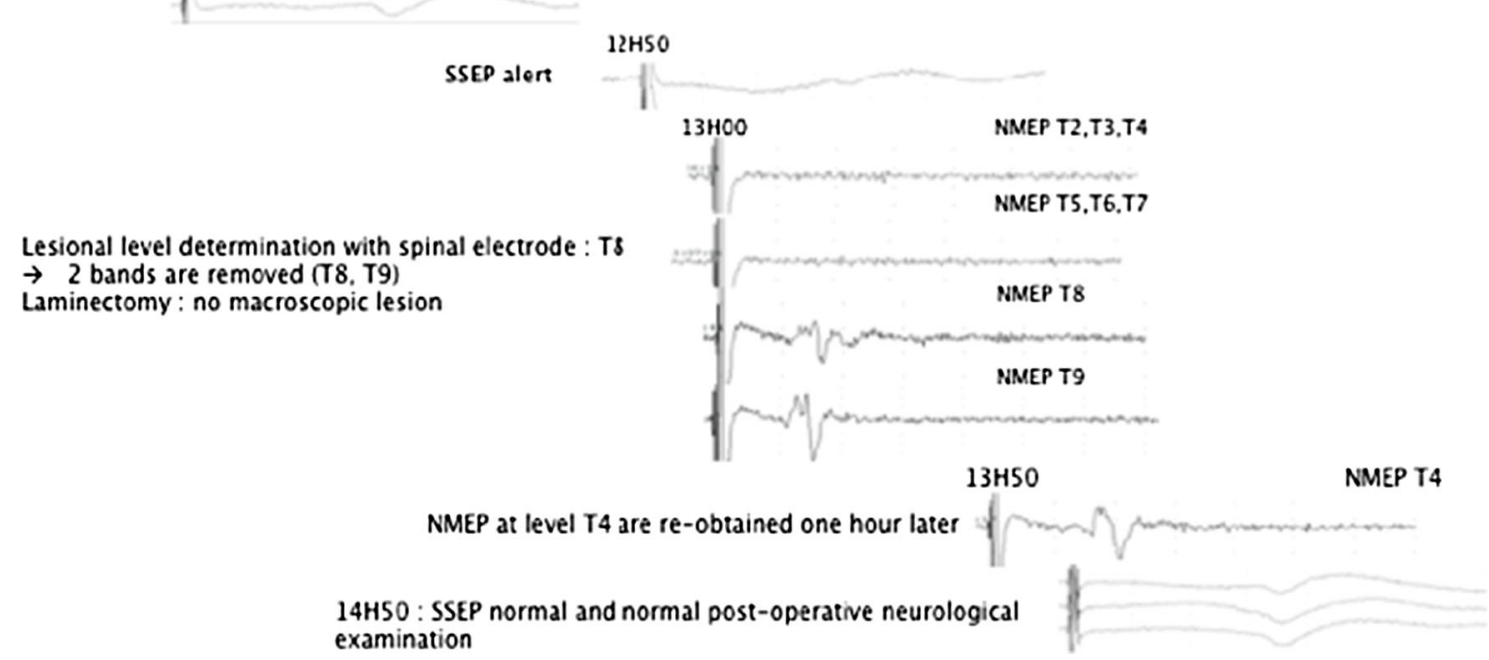

Fig. 1 Case report. Alerts in patient number 2. The anesthesiologic (hypertension 180/100 and bradycardia $50 \mathrm{bpm}$ ) and the neurologic alerts occur at $12 \mathrm{H} 50$. The lesional level was determined with the spinal electrode and localized at level T8. The bands T8T9 were

in this study. During the inclusion period, we continued to use other implants. This allowed us to make comparisons between different implants used in this period by the same surgeons of our team.

In the course of the 378 procedures, between 2006 and 2013, 15 out of 2,223 SB were involved and removed ( $0.67 \%$ of the sublaminar bands). Three out of 378 patients had post-operative neurologic deficiency, that is to say a neurologic complication rate of $0.8 \%$. One of them had a sensory suspended neurologic deficiency, that is to say a neurologic sequela rate of $0.2 \%$. This rate is not higher than the complication rate of other implants in the literature. Complications of pedicle screws are neurologic, vascular and pleural. Upendra et al. reported a rate of $10 \%$ of misplaced screws [8]. Herring reported a complication rate of $17 \%$ with Luque sublaminar wire [9]. In our series, we observed a comparable complication rate between SB (10 patients: $2.1 \%)$ and pedicular screws (7 patients: $1.8 \%)$. The other cause of neurologic alert is the correction removed and a laminectomy T9 was performed. NMEP were reobtained with a normal amplitude $1 \mathrm{~h}$ later. At the end of surgery, SSEP were normal. Post-operative neurological examination was normal

maneuver $(2.9 \%)$. The neurophysiologic alerts are distributed in the entire inclusion period, not only at the beginning of their use (Table 3). Other authors found no neurologic complication with SB within two different groups of 75 and 32 patients, and reported a satisfactory preservation of the correction in time [4, 5].

With their innocuousness, SB constitute a very interesting alternative to spine deviation surgery. Moreover, Blondel et al. have shown their superior capacity for sagittal balance restitution [10].

However, navigation spine surgery allows an increasingly reliable placement of pedicular screws, though it is at the initial stage of learning. We do not have this experience within our team.

SSEP and NMEP monitoring seems to be more discriminating when associated with an anesthesiologic monitoring with a continued invasive arterial blood pressure by catheterism. Indeed, in these alerts related to SB passage, neurophysiologic alerts are associated with 

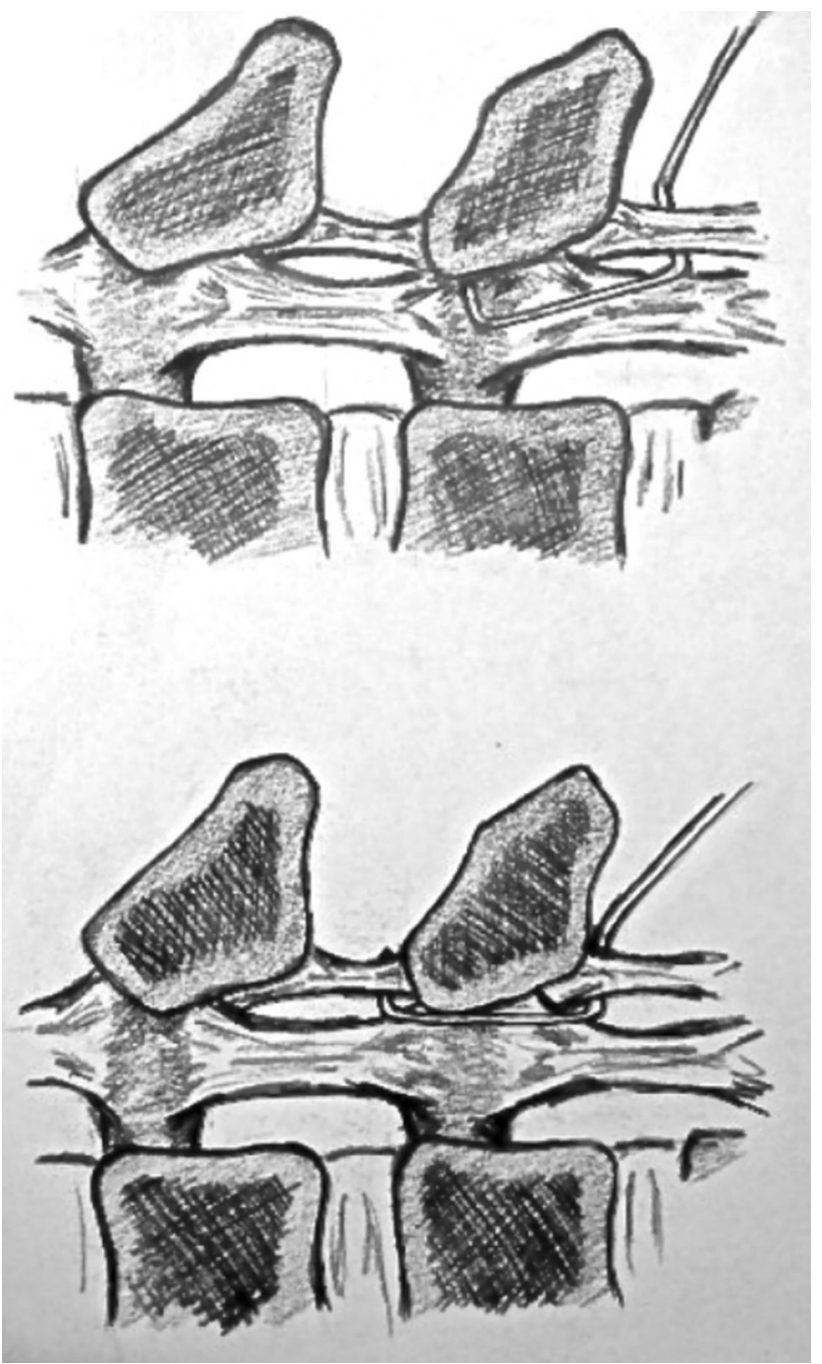

Fig. 2 The rectangular approach allows an atraumatic introduction avoiding a too deep penetration in the medullary canal

autonomic dysreflexia, including increased blood pressure and bradycardia (Fig. 1). However, this ten-case series is too limited to allow a correlation between the severity of hemodynamic alert and that of neurophysiologic alert. Persistent monitoring modification (unresolved alert) is associated with a post-operative neurologic deficiency (Table 4). Two neurophysiologic alerts were not associated with durable dysautonomic perturbations. These involved patients with no arterial catheterism. The lack of continued arterial blood pressure monitoring makes alert analysis difficult. When arterial blood pressure is not monitored on a continual basis, data collection is too spaced out, and if a very short autonomic dysreflexia occurs, it does not appear on the anesthesiologic diagram. In two cases, autonomic dysreflexia was concomittant with the neurophysiologic alert was consigned to the intra-operative neurophysiologic monitoring report.
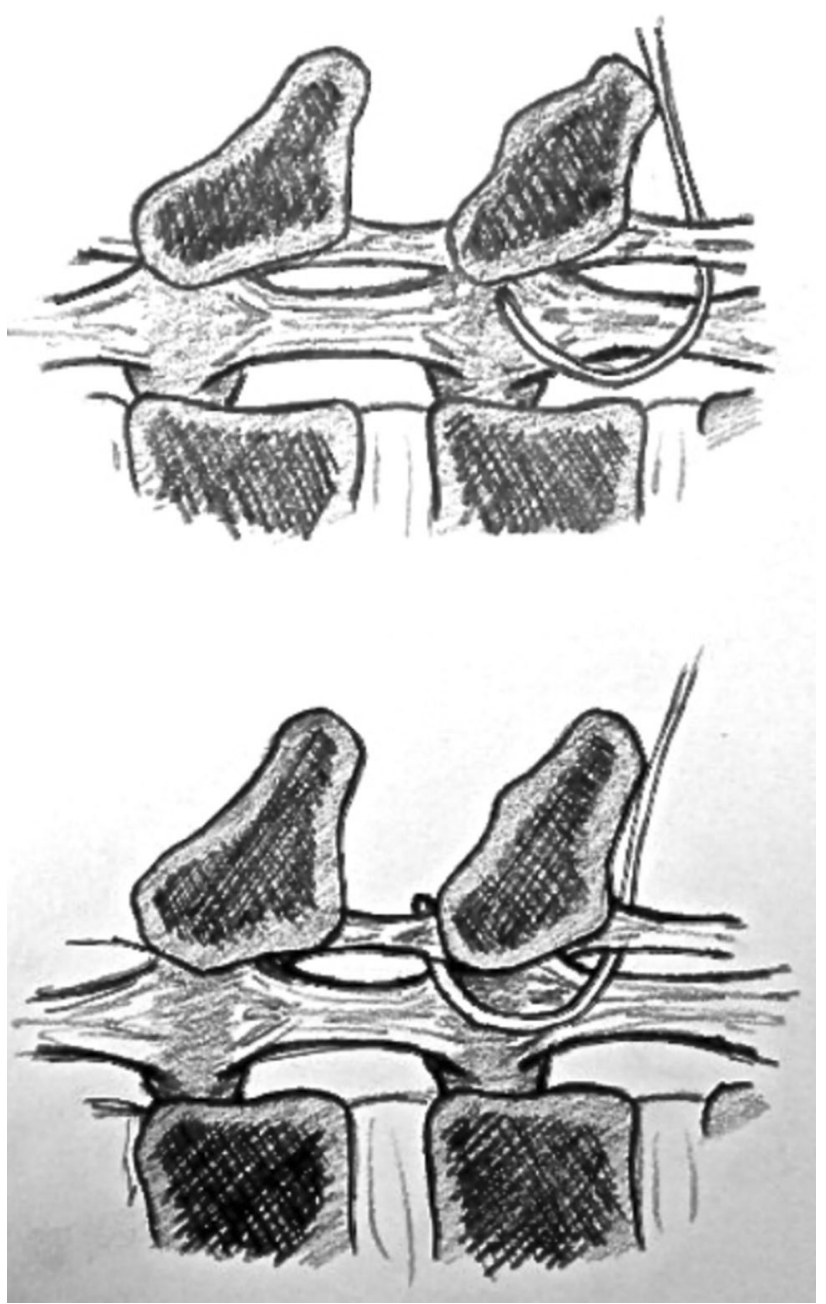

Fig. 3 If the curvature radius is too tight, the sublaminar band is introduced too deeply into the medullary canal and risks in cord injury

We also reviewed anesthesiologic and neurophysiologic monitoring of 21 patients who, at the time of the correction, had peri-operative alerts unrelated to SB. None of the 21 patients suffered from autonomic dysreflexia. There was no post-operative neurologic deficiency. This could suggest two different lesional mechanisms with two types of pathophysiology. Alerts due to sublaminar band passage suggest a traumatic lesional mechanism, like contusion or compression. This can be associated with a variable arterial spasm. This kind of traumatism can occur even if band introduction is not difficult for the surgeon. Alerts due to the reduction manoeuver seem to result from an arterial spasm of medullar vessels, which are very frail at the thoracic level. In the case of an ischemic mechanism, modifications of the neurophysiologic monitoring appeared about $20 \mathrm{~min}$ after the beginning of the correction [11].

This immediate acute arterial hypertension is compatible with a wide activation of the sympathetic system during spinal cord compression by the band passage. Animal 
models suggest that spinal cord compression gives an alpha-adrenergic mediated response (vasoconstriction of peripheral arterioles and increased venous return). The bradycardia may be explained by the baroreceptor response, induced by hypertension [12-14].

In that case, arterial hypertension must be respected to assure medullary perfusion pressure and medullary oxygenation, which are important factors of medullary protection. As a consequence, we strongly recommend a systematic complete equipment for every child spine deformity surgery, including arterial monitoring and neurophysiologic multimodal monitoring [15].

The group of patients with peri-operative alerts is comparable to the group of patients without alerts. Unlike to that in literature, risk factors such as male, secondary deformity and revision surgery, have not been found in this study [16].

The sublaminar band technique must focus on avoiding any medullary injury. The polymeric bands have a supple and malleable metallic band on one of their extremities. The surgeon must shape it manually to allow atraumatic passage on the Luque sublaminar wire mode [17]. To do this, we do not recommend wrapping it around the handle of a surgical instrument, as done sometimes. We recommend instead bending the metallic tip to give it a rectangular shape. In this way, the band will penetrate less deeply into the medullary canal during its passage under the lamina. The ideal shape for Luque wire has been described by many authors [18-21]. The band has to be more rectangular than circular (Figs. 2, 3). Its distal extremity is curved and during intracanal passage it must be in contact with the canal anterior face of the lamina, to avoid a dura injury. To prevent a penetration of SB that could be too deep during its passage under the lamina, the curvature radius applied to the band extremity should not be too tight. The ideal curvature radius corresponds to the width of the lamina under which the band is introduced. The extremity of the band is then grasped with a Kocher clamp at the upper edge of the lamina, to allow the passage of the entire band under the lamina.

\section{Conclusion}

When used appropriately, sublaminar bands are an effective and a safe alternative for spine deformity management. Their learning curve is simple and quick. The procedure must be performed with an anesthesiological arterial monitoring and a multimodal neurophysiologic monitoring, as with every spine deformity surgery. The implant preparation has to be meticulous to allow atraumatic passage under the lamina.
Acknowledgments We acknowledge Elisabeth Castanier for the statistics and Laurence Polirsztok for the English version.

\section{Conflict of interest None.}

Open Access This article is distributed under the terms of the Creative Commons Attribution License which permits any use, distribution, and reproduction in any medium, provided the original author(s) and the source are credited.

\section{References}

1. Hongo M, Ilharreborde B, Gay RE, Zhao C, Zhao KD, Berglund LJ, Zobitz M, An K (2009) Biomechanical evaluation of a new fixation device for the thoracic spine. Eur Spine J 18:1213-1219

2. Jouve JL, Meunier A, Ilharreborde B Traitement chirurgical de la scoliose par bandes polyesters sous lamaires: études précliniques et résultats cliniques

3. Jouve JL, Sales de Gauzy J, Blondel B, Launay F, Accabled F, Bollini G (2011) Use of the Universal Clamp for deformity correction and as an adjunct to fusion: preliminary results in scoliosis. J Child Orthop 4:73-80

4. Sales de Gauzy J, Jouve JL, Accadbled F, Blondel B, Bollini G (2011) Use of the Universal Clamp in adolescent idiopathic scoliosis for deformity correction and as an adjunct to fusion: 2-year follow-up. J Child Orthop 5:273-282

5. Mazda K, Ilharreborde B, Even J, Lefevre Y, Fitoussi F, Penneçot G (2009) Efficacy and safety of posteromedial translation for correction of thoracic curves in adolescent idiopathic scoliosis using a new connection to the spine: the Universal Clamp. Eur Spine J 18:158-169

6. Gavaret M, Jouve JL, Péréon Y, Accadbled F, André-Obadia N, Azabou E, Blondel B, Bollini G, Delécrin J, Farcy JP, FournetFayard J, Garin C, Henry P, Manel V, Mutschler V, Perrin G, Sales de Gauzy J, the French Society of Spine Surgery (SFCR) (2013) Intraoperative neurophysiologic monitoring in spine surgery. Developments and state of the art in France in 2011. Orthop Traumatol Surg Res

7. Accadbled F, Henry P, Sales de Gauzy J, Cahuzac JP (2006) Spinal cord monitoring in scoliosis surgery using an epidural electrode. Results of a prospective, consecutive series of 191 cases. Spine 22:2614-2623

8. Uprenda BN, Meena D, Chowdhury B, Ahmad A, Jayaswal A (2008) Outcome-based classification for assesment of thoracic pedicular screw placement. Spine 33:384-390

9. Herring JA, Wenger DR (1982) Segmental spinal instrumentation. A preliminary report of 40 consecutive cases. Spine 3:285-298

10. Blondel B, Lafage V, Farcy JP, Schwab F, Bollini G, Jouve JL (2012) Influence of screw type on initial coronal and sagittal radiological correction with hybrid constructs in adolescent idiopathic scoliosis. Correct Prior OTSR 98:873-878

11. Kai Y, Owen J, Lenke LG et al (1993) Use of sciatic neurogenic motor evoked potentials versus spinal potentiels to predict earlyonset neurologic deficits when intervention is still possible during overdistraction. Spine 18:1134-1139

12. Eidelberg ED (1973) Cardiovascular response to experimental spinal cord compression. J Neurosurg 38(3):326-331

13. Rawe SE, Perot PL Jr (1979) Pressor response resulting from experimental contusion injury to spinal cord. J Neurosurg 50(1):58-63

14. Young W, DeCrescito V, Tomasula JJ, Ho V (1980) The role of the sympathetic nervous system in pressor responses induced by spinal injury. J Neurosurg 52(4):473-481 
15. Ambardekar AP, Sestokas AK, Schwartz DM, Flynn JM, Rehman M (2010) Concomitant hypertension, bradycardia, and loss of transcranial electric motor evoked potentials during pedicle hook removal: report of a case. J Clin Monit Comput 24:437-440

16. Gavaret M, Trébuchon A, Aubert S, Jacopin S, Blondel B, Glard Y, Jouve JL, Bollini G (2011) Intraoperative monitoring in pediatric orthopedic spinal surgery: three hundred consecutive monitoring cases of which $10 \%$ of patients were younger than 4 years of age. Spine 22:1855-1863

17. Luque ER (1982) The anatomic basis and development of segmental spinal instrumentation. Spine 3:256-259
18. Zindrick MR, Knight GW, Bunch WH, Miller MC, Butler DM, Lorenz M, Behal R (1989) Factors influencing the penetration of wires into the neural canal during segmental wiring. JBJS 5:742-750

19. Kim D, Vaccaro A (2005) Spinal instrumentation: surgical techniques. Chap 81

20. McMaster MJ (1991) Luque rod instrumentation in the treatment of adolescent idiopathic scoliosis. JBJS 73-B:982-989

21. Fergusson RL (1994) L rod instrumentation. The pediatric spine: principles and practice. In: Weinstein SL (ed), Chap 78, pp 1659-81 